\title{
What determines large scale galaxy clustering: halo mass or local density?
}

\author{
Arnau Pujol ${ }^{1}$, Kai Hoffmann ${ }^{1}$, Noelia Jiménez ${ }^{1,2}$, and Enrique Gaztañaga ${ }^{1}$ \\ 1 Institut de Ciències de l'Espai (ICE, IEEC/CSIC), 08193 Bellaterra (Barcelona), Spain \\ e-mail: arnaupv@gmail.com \\ 2 School of Physics \& Astronomy, University of St Andrews, North Haugh, St Andrews KY16 9SS, Scotland, UK \\ Received 15 June 2016 / Accepted 13 October 2016
}

\begin{abstract}
Using a dark matter simulation we show how halo bias is determined by local density and not by halo mass. This is not totally surprising as, according to the peak-background split model, local matter density $(\bar{\delta})$ is the property that constrains bias at large scales. Massive haloes have a high clustering because they reside in high density regions. Small haloes can be found in a wide range of environments which differentially determine their clustering amplitudes. This contradicts the assumption made by standard halo occupation distribution (HOD) models that bias and occupation of haloes is determined solely by their mass. We show that the bias of central galaxies from semi-analytic models of galaxy formation as a function of luminosity and colour is therefore not correctly predicted by the standard HOD model. Using $\bar{\delta}$ (of matter or galaxies) instead of halo mass, the HOD model correctly predicts galaxy bias. These results indicate the need to include information about local density and not only mass in order to correctly apply HOD analysis in these galaxy samples. This new model can be readily applied to observations and has the advantage that, in contrast with the dark matter halo mass, the galaxy density can be directly observed.
\end{abstract}

Key words. dark matter - large-scale structure of Universe

\section{Introduction}

In the standard cosmological framework, the so-called $\Lambda$ cold dark matter $(\Lambda \mathrm{CDM})$ paradigm, galaxies form, evolve and reside in dark matter haloes that grow and assemble in a hierarchical way (White \& Rees 1978). Therefore, it is assumed that the intrinsic properties and the evolution of these host haloes will have an impact in the subsequent galaxy population inhabiting the haloes. The unknown nature of the dark matter (and dark energy) could be unveiled through the study of the baryonic observables (such as galaxies) once we properly understand their co-evolution, and this task, modelling the relation between the observed galaxy distribution and the underlying dark matter field, is a fundamental question of modern cosmology.

Given a cosmology, the halo mass function, the halo concentration and halo bias can be defined. This implies that the dark matter density field is not perfectly mapped by the distribution of the dark matter haloes, since they are biased tracers of it. In order to model the galaxy clustering statistics, it is necessary to specify the number and spatial distribution of galaxies within these dark matter haloes. One simple approach is to use the statistical halo distribution, known as the standard halo occupation distribution (HOD; Jing et al. 1998; Benson et al. 2000; Seljak 2000; Scoccimarro et al. 2001; Cooray \& Sheth 2002; Berlind \& Weinberg 2002). Its simplicity resides in the common assumption that the derived occupation parameters and the physical properties of the galaxies are solely determined by the mass of the halo in which they reside. Therefore, the probability that a halo of mass $m$ hosts $N_{\text {gal }}$ galaxies of a given property is given by the quantity $P\left(N_{\text {gal }} \mid m\right)$.

The HOD has been proven a very powerful theoretical tool to constrain both the galaxy-halo connection and the fundamental parameters in cosmology (Yang et al. 2003; Zehavi et al. 2005, 2011; Cooray 2006; van den Bosch et al. 2007; Zheng et al. 2007; Tinker et al. 2013). Through the use of the HOD model, mock galaxy catalogues are constructed for studies of galaxy formation as well as for the preparation and analysis of observational surveys (Berlind \& Weinberg 2002; Zheng et al. 2007; Rodríguez-Torres et al. 2016; Carretero et al. 2015). It has become clear, however, that the clustering of dark matter haloes depends on other properties besides mass (Sheth \& Tormen 2004; Gao et al. 2005; Conroy et al. 2006; Gao \& White 2007; Jing et al. 2007; Wetzel et al. 2007; Faltenbacher \& White 2010; Lacerna \& Padilla 2011; Lacerna et al. 2014; Pujol \& Gaztañaga 2014). For example, the construction of mock galaxy catalogues through $\mathrm{N}$-body simulations hasshown that the amplitude of the two-point correlation function of dark matter haloes with masses lower than $10^{13} h^{-1} M_{\odot}$ on large scales depends on the halo formation time (e.g., Gao et al. 2005). Additionally, halo properties such as concentration, shape, halo spin, major merger rate, triaxiality, shape of the velocity ellipsoid and velocity anisotropy, show correlations with properties other than halo mass (Bett et al. 2007; Croton et al. 2007; Lacerna \& Padilla 2012). Moreover, haloes of equal mass could have different galaxy occupation statistics depending on their environment (e.g., Croft et al. 2012; Pujol \& Gaztañaga 2014). Ignoring the effects of properties other than mass in the HOD modelling could distort the conclusions and interpretations of the observational results (Zentner et al. 2014).

To solve this issue, new studies point in the direction of taking into account these halo properties linked with the environment and their formation history and modifying the standard HOD accordingly. The relevant properties can be incorporated into the formulation of more flexible schemes of HOD used to produce mock catalogues comparable with observations. Such catalogues have recently been presented by Croton et al. (2007) and Masaki et al. (2013), who introduced a rank ordering of galaxy colours or star formation rate (SFR). The authors point out that, for fixed halo mass, red galaxies are more clustered 
than blue galaxies. These, together with the "age-matching" models of Hearin et al. (2013, 2014), have been shown to successfully reproduce a number of observed signals such as the two-point clustering and galaxy-galaxy lensing signal of SDSS galaxies (Lacerna et al. 2014, and references therein). Recently, Hearin et al. (2016) presented the idea of decorated HOD, a new parametrisation that allows the galaxy sample to be affected by a parametrised assembly bias.

According to the peak-background split model (Bardeen et al. 1986; Cole \& Kaiser 1989), the fundamental quantity that describes halo bias is the density fluctuation of the dark matter field, and not mass. Hence, we expect local density to constrain bias better than mass. In this paper we show the dependence of large scale halo bias on mass and local density (defined as the fluctuations of the local background density $\bar{\delta}$ ), and confirm the peak-background split prediction that density is the property that constrains bias.

The aim of our study is to analyse how well mass and local density determine galaxy bias and HOD. For this, we present a bias reconstruction method that tests how well the standard HOD assumptions (that halo bias and occupation depend only on mass) are able to correctly predict galaxy bias. To do this, we compare the measured galaxy bias with the one reconstructed from the measurements of halo bias and HOD. This method has already been implemented in Pujol \& Gaztañaga (2014), where we showed this test for luminosity dependent galaxy bias. In this paper, we also use the reconstruction method to test how well local density is able to predict galaxy bias from halo bias and HOD. We find that mass is not able to predict galaxy bias for colour-selected samples and that, in these cases, local density makes a much better prediction.

Our analysis is based on haloes from the Millennium simulation (Springel et al. 2005) and the semi-analytical model (SAM) of galaxy formation of Guo et al. (2011). The SAM populate haloes with galaxies that are evolved and followed in time inside the complex structure of merger-trees. The baryonic processes included are laws for metal-dependent gas cooling, reionization, star formation, gas accretion, merging, disk instabilities, AGN and supernovae feedback, ram pressure stripping and dust extinction, among others (e.g., Baugh 2006; Jiménez et al. 2011; Gargiulo et al. 2015). Because of these processes, the resulting galaxy population produced by SAMs is sensitive to the environment and evolution of dark matter haloes. Depending on the galaxy selection, the information from halo mass alone could be insufficiently correlated with the clustering of galaxies. In these cases, local density is more useful for the determination of galaxy bias than halo mass. Thus, we propose to use the information from local density to improve the HOD analyses on surveys and theory.

The paper is organised as follows. In Sect. 2 we describe the data used and the methodology for our measurements of clustering, bias and for our HOD reconstructions. The results are shown in Sect. 3, and we summarise the conclusions of the paper in Sect. 4.

\section{Methodology}

\subsection{Simulation data}

In this study we use the data from the Millennium simulation $^{1}$ (Springel et al. 2005), an $N$-body simulation generated using the GADGET-2 code (Springel 2005). The simulation corresponds to a $\Lambda$-CDM cosmology with the following

\footnotetext{
1 http://wWW.mpa-garching.mpg.de/millennium/
}

parameters: $\Omega_{\mathrm{m}}=0.25, \Omega_{b}=0.045, h=0.73, \Omega_{\Lambda}=0.75$, $n=1$ and $\sigma_{8}=0.9$. It contains $2160^{3}$ particles in a comoving box with a side length of $500 \mathrm{~h}^{-1} \mathrm{Mpc}$. The resolution corresponds to a particle mass of $8.6 \times 10^{8} h^{-1} M_{\odot}$ and a spatial resolution of $5 h^{-1} \mathrm{kpc}$. The cosmological model is based on WMAP1 (Spergel et al. 2003) and the 2-degree Fields Galaxy Redshift Survey (2dFGRS) data (Cole et al. 2005). The initial conditions were calculated using CMBFAST (Seljak \& Zaldarriaga 1996). We use the comoving output at $z=0$.

The haloes are identified using the friends-of-friends (FOF) algorithm, using a linking length of 0.2 times the mean particle separation, and discarding all haloes with less than 20 particles. In our analysis we define the halo mass from the total number of particles belonging to the FOFs.

Galaxy catalogues of several SAM are available in the public database of the simulation. For this analysis we used four models (Bower et al. 2006; De Lucia \& Blaizot 2007; Font et al. 2008; Guo et al. 2011). Although the results are different for each model, they all show the same behaviours and the conclusions of our study do not depend on the SAM used. For this, we show the results from Guo et al. (2011) in Sect. 3, and we show a comparison of the rest of the models in Appendix A.

\subsection{Clustering and bias}

Spatial fluctuations of the matter or tracer density $\rho$ are defined as normalised deviations from the mean density $\bar{\rho}$ at the position $r$, that is,

$\delta(\boldsymbol{r}) \equiv \frac{\rho(\boldsymbol{r})-\bar{\rho}}{\bar{\rho}}$.

Note that in this article we often refer to these density fluctuations as density for simplicity. We measure $\delta(\boldsymbol{r})$ by dividing the simulation into cubical grid cells with side lengths of $500 / 64 \sim 8 h^{-1} \mathrm{Mpc}$ and assigning a $\delta$ to each cell. We then measure the two-point correlation function as

$\xi_{\mathrm{AB}}(r) \equiv\left\langle\delta_{\mathrm{A}}\left(\boldsymbol{r}_{1}\right) \delta_{\mathrm{B}}\left(\boldsymbol{r}_{2}\right)\right\rangle$,

which is a function of the scale $r \equiv\left|\boldsymbol{r}_{2}-\boldsymbol{r}_{1}\right|$. The average $\langle\ldots\rangle$ is taken over all pairs of $\delta$ in the analysed volume, independently of their orientation. The indices A and B refer to the fluctuations of different density tracers (here haloes or galaxies, which we generically call $\delta_{\mathrm{G}}$ ) or to those of the matter density, which we denote as $\delta_{\mathrm{m}}$. Hence, $\mathrm{A}=\mathrm{B}=\mathrm{m}$ denotes the matter autocorrelation $\left(\xi_{\mathrm{mm}}\right)$, while $\xi_{\mathrm{Gm}}$ is the cross-correlation between the tracer $\mathrm{G}$ and matter $\mathrm{m}$. We then estimate the bias by the ratio:

$b(r) \equiv \frac{\xi_{\mathrm{Gm}}(r)}{\xi_{\mathrm{mm}}(r)}$.

At large scales $\left(r \gtrsim 20 h^{-1} \mathrm{Mpc}\right)$, where $\xi<1$, this ratio is well described by a constant, which is in good agreement with the linear bias and tends to the value $\delta_{G} \simeq b_{1} \delta_{\mathrm{m}}$, (e.g. see Bel et al. 2015). We estimate this linear bias $b_{1}$, which we refer to as $b$, by fitting $b(r)$ with a constant in the scale range of $20 h^{-1} \mathrm{Mpc}<r<30 h^{-1} \mathrm{Mpc}$. For larger scales, the measurements start to be noisy due to the size of the simulation. The covariance of these measurements is derived by jack-knifing (e.g. Norberg et al. 2009) using 64 cubical subvolumes.

The value of $b$ depends on how the tracers are selected. In galaxy surveys, the limits of the observed galaxy luminosities roughly correspond to a selection by host halo mass. The 


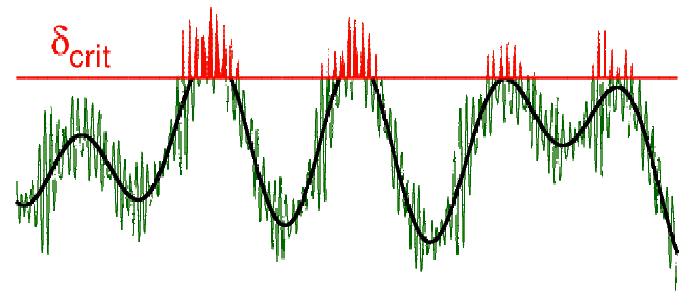

Fig. 1. Illustration of peak-background split model, $\delta=\delta_{\mathrm{s}}+\bar{\delta}$, showing the location of haloes that form above some critical value $\delta_{\text {crit }}$ in red. The clustering of those haloes is stronger than the rest (in green). But note how once the local density $\bar{\delta}$ (black curve) is fixed at $\delta_{\text {crit }}$, the largescale clustering of haloes (red) does not depend on the peak-hight (halo mass).

mass dependence of the bias can be predicted from the peakbackgroundsplit model (see Bardeen et al. 1986; Cole \& Kaiser 1989; Mo \& White 1996; and Hoffmann et al. 2015, for a recent validation of the predictions) where the matter density field is described by the superposition of small-scale fluctuations (peaks) $\delta_{\mathrm{s}}$ with large-scale fluctuations of the background matter density $\bar{\delta}$ around each peak (i.e. $\delta=\delta_{\mathrm{s}}+\bar{\delta}$ ). In this model background fluctuations can lift the peak-heights above a critical density $\delta_{\text {crit }}$ at which they collapse to haloes as illustrated by Fig. 1. The mass of these haloes then corresponds to the peak-heights. Consequently massive haloes tend to reside in environments where the background density is high, while low-mass haloes can reside in a broader range of environmental densities (we show this effect with haloes from the Millennium simulation in Sect. 3.1). Massive haloes are therefore more strongly clustered than low-mass haloes, which causes a mass dependence of the bias parameter.

\subsection{Local background density}

The bias of a given halo sample can also depend on additional halo properties besides the halo mass, such as the concentration of the mass density profile or the properties of the galaxies hosted by the halo (see e.g. Gao et al. 2005; Faltenbacher \& White 2010; Hearin et al. 2015). These additional properties can be related to the merging history of haloes and lead to the so-called assembly bias. Furthermore, the bias of a given halo sample is affected by the tidal forces from the large-scale environment, which is known as non-local bias (see Chan et al. 2012; Baldauf et al. 2012; Bel et al. 2015).

These effects can lead to false predictions by models that rely on the assumption that the mass of a halo sample completely determines the bias, such as self-calibration techniques (e.g. Wu et al. 2008) or HOD models (Zentner et al. 2014; Pujol \& Gaztañaga 2014). A way to circumvent problems for the HOD model is to use a halo property different from the mass, which completely determines the bias of a given halo population. The peak-background split argument described in Sect. 2.2 suggests that the clustering of peaks (which corresponds to tracers such as haloes) is determined by fluctuations of the large-scale background density $\bar{\delta}$. Hence, for fixed background densities, the bias should be independent of the tracer properties (see Fig. 1 for illustration).

To obtain a visual impression of this argument, in Fig. 2 we show the spatial distribution of haloes that reside in regions with different densities, defined by the value of $\bar{\delta}$ estimated around each halo smoothed on cubical cells of side $l=14 h^{-1} \mathrm{Mpc}$ as explained below. By comparing the four panels of the figure one can see that the large-scale clustering, and hence the bias,

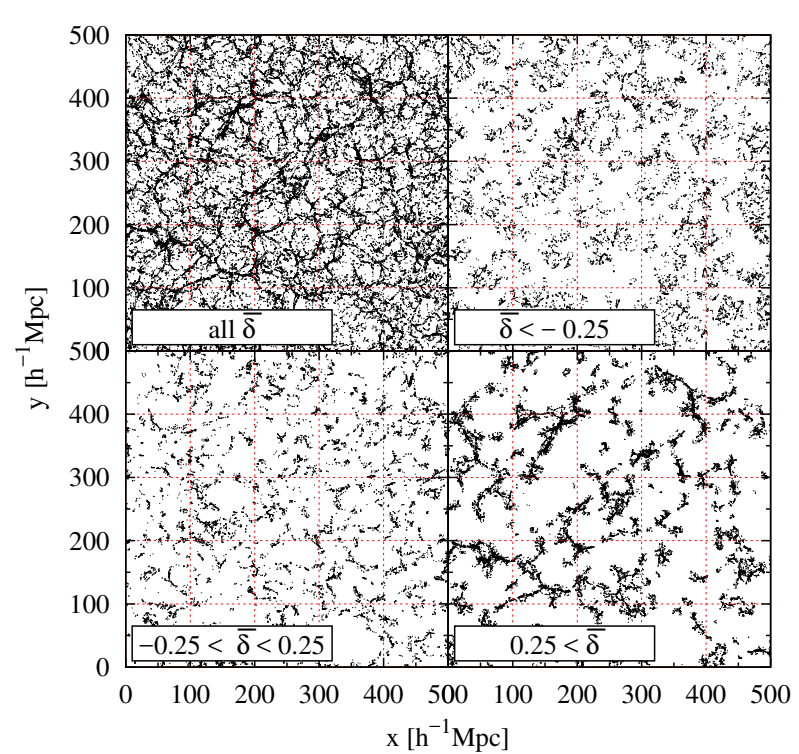

Fig. 2. Distribution of haloes with different dark matter local densities $\bar{\delta}$ in a $2 h^{-1} \mathrm{Mpc}$ slice of the Millennium simulation. The top left panel shows all haloes, while the top right shows haloes in under-densities. The bottom left shows haloes with mean background densities while the bottom right shows haloes in over-dense regions.

strongly changes with the local dark matter back-ground density $\bar{\delta}$ (as we show in Sect. 3.2). On the other hand, the large-scale clustering is nearly independent of halo mass (not shown in this figure) when haloes are selected by $\bar{\delta}$. Note that a weak mass dependence of the bias for fixed $\bar{\delta}$ can be expected from the aforementioned non-local bias.

When determining $\bar{\delta}$ around a given halo, we face the problem that the dark matter density distribution of the Millennium simulation is only publicly available as a 500/256 $\simeq 2 h^{-1} \mathrm{Mpc}$ grid. We therefore assigned the density in a cubical volume around each halo, with length $l \simeq 6,14$ and $26 h^{-1} \mathrm{Mpc}$, which have the same volume as a sphere of radius $R=3.72,8.68$ and $16.13 h^{-1} \mathrm{Mpc}$, respectively. The position of these volumes has a $2 h^{-1} \mathrm{Mpc}$ inaccuracy, which results from the grid cell size. In the presentation of our results we focus on the intermediate scale $R=8.68 h^{-1} \mathrm{Mpc}$, while this choice does not affect our conclusion, as results for the other scales are similar.

The linear halo bias $b_{\mathrm{h}}$ is shown as a function of $\bar{\delta}$ in Fig. 3 using haloes of all masses. The bias increases with the density of the environment and becomes negative in under-dense regions. Note that we find negative bias because we study the halo-matter cross-correlation, while in the case of the auto-correlation the bias would remain positive. The dependence of $b_{\mathrm{h}}$ on $\bar{\delta}$ becomes weaker when $\bar{\delta}$ is defined at smaller scales $R$. Note that the clustering of galaxy with different environmental densities has been studied in data by Abbas \& Sheth (2007).

Since the large-scale clustering of the tracers corresponds to the clustering of the background density fluctuations in which they reside, we can compare our measurements to predictions for the clustering of the excursion set model (Sheth 1998). We use Eq. (5) from Abbas \& Sheth (2007):

$b \simeq \frac{\bar{\delta}_{0}}{\sigma_{0}^{2}}$,

where $\bar{\delta}_{0}$ is the initial linear over-density and $\sigma_{0}$ is the corresponding linear variance on the Lagrangian scale $R_{0}=R(1+$ $\bar{\delta})^{1 / 3}$. To estimate $\bar{\delta}_{0}$, we use the spherical collapse model to 


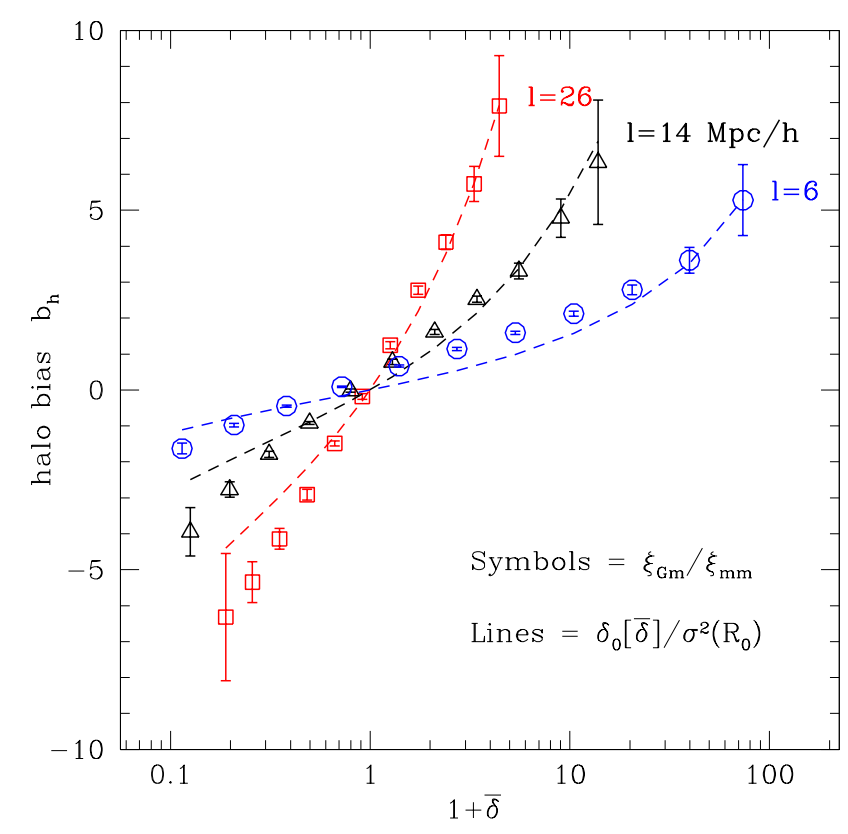

Fig. 3. Halo bias $b_{\mathrm{h}}$ as a function of mass local density $\bar{\delta}$ in cubical volumes with side length $l=26 h^{-1} \mathrm{Mpc}$ (red), $l=14 h^{-1} \mathrm{Mpc}$ (black) and $l=6 h^{-1} \mathrm{Mpc}$ (blue), which have the same volume as a sphere of radius $R=3.72,8.68$ and $16.13 h^{-1} \mathrm{Mpc}$, respectively. The corresponding dashed line shows a simple prediction $b \simeq \delta_{0} / \sigma_{0}^{2}$ where $\bar{\delta}_{0}$ is the linear density corresponding to $\bar{\delta}$ (according to the spherical collapse model) and $\sigma_{0}^{2}$ is the linear variance at Lagrangian scale $R_{0}=R(1+\bar{\delta})^{1 / 3}$.

map the measured non-linear local density into the initial density (see also Fosalba \& Gaztanaga 1998). These predictions are in general in good agreement with the measurements and do not include any free parameters. Note that some of the differences at small $\bar{\delta}$ could come from the fact that we measure the bias in bins of $\bar{\delta}$, while the prediction are for threshold values of $\bar{\delta}_{0}$, so we expect them to be systematically higher. This can easily be corrected, but is beyond the scope of this paper.

In the following subsection we will directly use these bias measurements $b_{\mathrm{h}}(\bar{\delta})$ to study the bias predictions from the standard HOD assumptions, and to present a new HOD test based on local density $\bar{\delta}$, which is less affected by assembly bias.

\subsection{Mass and density $H O D$ bias reconstruction}

The standard HOD model is based on the assumption that halo bias is determined solely by the halo mass $m$ of the sample. We will therefore refer to it as the mHOD model in the following. In this mHOD model, the bias of galaxies $\left(b_{\mathrm{g}}\right)$, which are selected by an arbitrary property $P$, are reconstructed from the halo bias as a function of mass $b_{\mathrm{h}}(\mathrm{m})$ and the mean number of galaxies with property $P$ per halo with mass $m,\left\langle N_{\mathrm{g}}(m \mid P)\right\rangle$ as

$b_{\text {rec }}^{m}(P)=\frac{\int \mathrm{d} m b_{\mathrm{h}}(m)\left\langle N_{\mathrm{g}}(m \mid P)\right\rangle}{\int \mathrm{d} m\left\langle N_{\mathrm{g}}(m \mid P)\right\rangle}$.

This equation can be seen as a weighted average of $b_{\mathrm{h}}(m)$, where the weight is given by $\left\langle N_{\mathrm{g}}(m \mid P)\right\rangle$. The mHOD model provides a way to infer the average mass of host haloes in which a given galaxy population is residing by varying $\left\langle N_{\mathrm{g}}(m \mid P)\right\rangle$ in order to reproduce the observed bias, that is, $b_{\mathrm{rec}}^{m}(P)=b_{\mathrm{g}}(P)$. However, this relies on the assumption that the bias of a galaxy population selected by the property $P$ is completely determined by their host halo mass, which might not be correct as discussed in Sect. 2.2.
Cosmological $N$-body simulations allow us to test the mHOD model, as we can measure $b_{\mathrm{h}}(m)$ and $\left\langle N_{\mathrm{g}}(m \mid P)\right\rangle$ and compare the predicted $b_{\text {rec }}^{m}(P)$ to measurements of $b_{\mathrm{g}}(P)$. This test was explored by Pujol \& Gaztañaga (2014) and will hereafter be referred to as the bias reconstruction method. The test revealed that the mHOD model fails to predict the bias correctly in the low halo mass rangebut that it works with 5-10\% bias accuracy in the high mass end.

In the previous subsection we considered the redefinition of the HOD model using the local background density $\bar{\delta}$ around a given halo instead of the halo mass. This approach has the advantage that $\bar{\delta}$ is expected to determine the bias of a given halo or galaxy population better than the mass (we study this in Sect. 3). Furthermore, $\bar{\delta}$ is well defined, whereas the halo mass can vary significantly, depending on the definition. In this paper we refer to the density HOD (dHOD) model (using density), analogous to the mHOD model (using mass). The corresponding bias reconstruction is obtained simply by replacing the halo mass in Eq. (5) by $\bar{\delta}$,

$b_{\mathrm{rec}}^{\bar{\delta}}(P)=\frac{\int \mathrm{d} \bar{\delta} b_{\mathrm{h}}(\bar{\delta})\left\langle N_{\mathrm{g}}(\bar{\delta} \mid P)\right\rangle}{\int \mathrm{d} \bar{\delta}\left\langle N_{\mathrm{g}}(\bar{\delta} \mid P)\right\rangle}$.

In the following section we test how well our new dHOD model predicts the bias compared to the standard mHOD model.

\section{Results}

\subsection{Halo bias}

In this subsection we analyse the dependence of halo bias on halo mass and local background matter density fluctuations $\bar{\delta}$ (smoothed with a cubical top hat filter with a side length of $l=14 h^{-1} \mathrm{Mpc}$, which has the same volume as a sphere of radius $R=8.68 h^{-1} \mathrm{Mpc}$ ) in the environment of each halo. The measurement of the latter is described in Sect. 2. Similar results are found for other smoothing scales.

In the left panel of Fig. 4 we show the halo bias $\left(b_{\mathrm{h}}\right)$, measured via Eq. (3), as a function of the halo mass $m$ for haloes within different $\bar{\delta}$ ranges. The black line corresponds to $b_{\mathrm{h}}(m)$ for all haloes, selected independently of $\bar{\delta}$. This latter measurement is consistent with the theoretical model of Tinker et al. (2010; see Fig. 3 of Pujol \& Gaztañaga 2014). We can see that $b_{\mathrm{h}}(m)$ does not change significantly (less than a $10 \%$ ) with halo mass when $\bar{\delta}$ is fixed, while it changes significantly when all the haloes are included (by approximately a factor of 2 in the range of masses shown here). In the right panel we show the same analysis from a different point of view. Here we present $b_{\mathrm{h}}(\bar{\delta})$ for different $m$ bins. Each line corresponds to a range in $m$, while the black solid line shows $b_{\mathrm{h}}(\bar{\delta})$ for all the haloes. We can see a strong dependence of $b_{\mathrm{h}}$ on $\bar{\delta}$, and that $b_{\mathrm{h}}(\bar{\delta})$ depends weakly on $m$.

These measurements demonstrate the argument from the peak-background split that local density is the property that constrains clustering. We show that the bias of a given halo sample is well constrained when the haloes are solely selected by the local density in their environment $\bar{\delta}$, almost independently of the halo mass.

If $\bar{\delta}$ is left as a free parameter, a mass dependence of the bias arises from the fact that high mass haloes tend to reside in high density regions and low mass haloes in regions with lower density. This tendency can be seen in the central panel of Fig. 5, where we show the mass versus the local density $\bar{\delta}$ for each halo in the simulation. The colours describe the number of haloes 

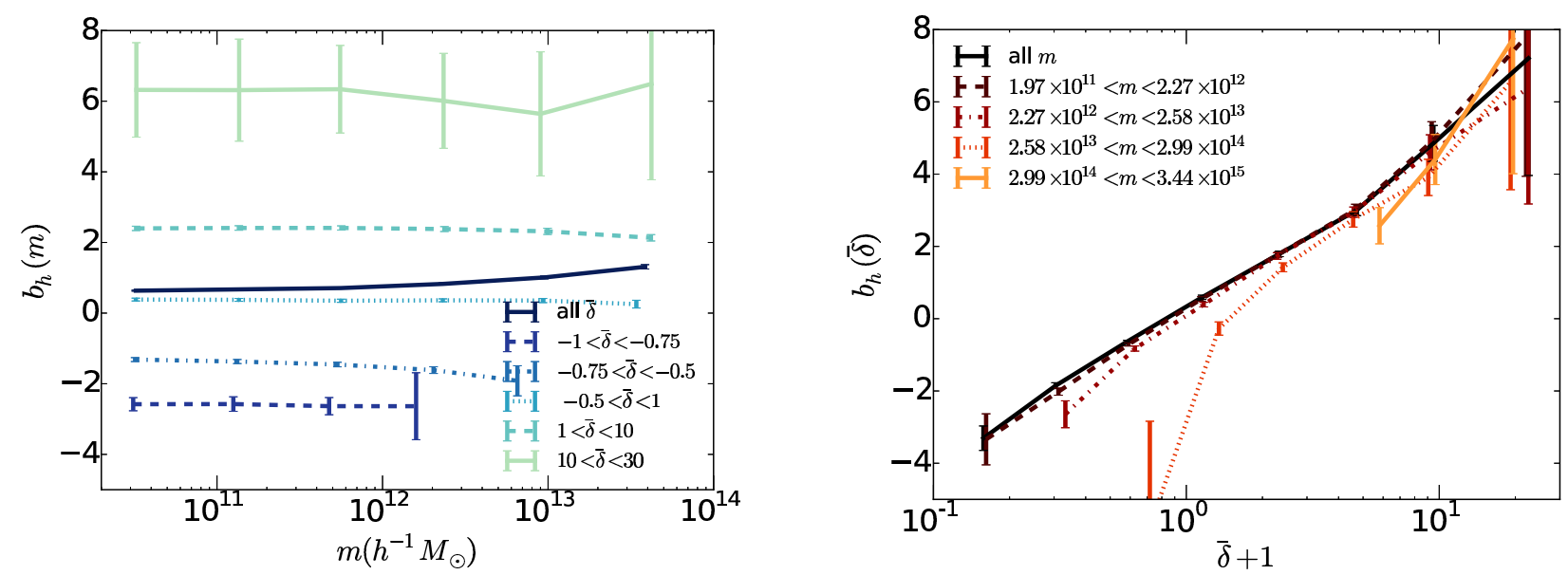

Fig. 4. Left: halo bias as a function of mass. Each line corresponds to haloes in a fixed density fluctuations $\bar{\delta}$. Right: halo bias as a function of the local density $\bar{\delta}$. Each line corresponds to a different halo mass bin. In both panels, $\bar{\delta}$ is defined as the Eulerian density fluctuation around a cubic box of $14 h^{-1} \mathrm{Mpc}$ of side.

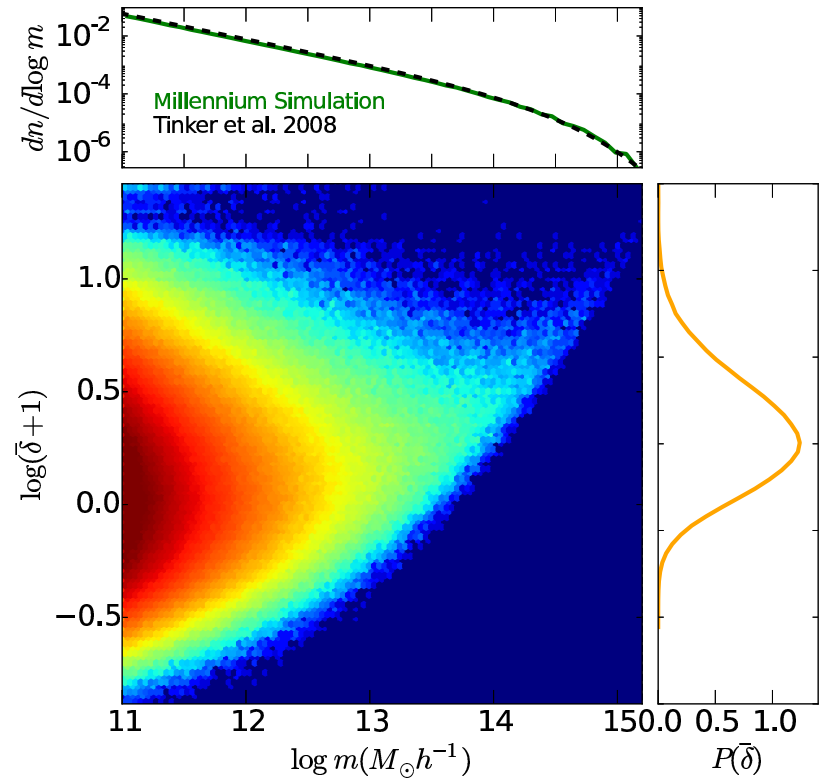

Fig. 5. Distribution of haloes in local density $\bar{\delta}$ and mass $m$. The colours show the number of haloes that are in a density $\bar{\delta}$ and have a mass $m$. Right panel shows the $\bar{\delta}$ distribution from the contribution of all the haloes, $P(1+\bar{\delta})$. The top panel shows the Halo Mass Function.

with the corresponding mass and density. By integrating this distribution over $\bar{\delta}$ we derive the HMF, $\left\langle N_{\mathrm{h}}(m, \bar{\delta}) /(V \log (m))\right\rangle_{\bar{\delta}}$ (where $V$ is the simulation volume and $N_{\mathrm{h}}$ the absolute number of haloes). The HMF is well described by the Tinker et al. (2008) model, as shown in the top panel of Fig. 5. By integrating over the mass $m$, we obtain the probability distribution function (PDF) of $\bar{\delta}$, or $\left\langle N_{\mathrm{h}}(\bar{\delta}, m) / n(m)\right\rangle_{\mathrm{m}}$, which is roughly log-normal, as shown in the right panel of Fig. 5.

The aforementioned tendency, that high mass haloes tend to reside in high density regions and low mass in regions with lower density, can be seen more clearly in Fig. 6. In the top panel of this figure we show the HMF of haloes with different background densities $\bar{\delta}$. We find that the fraction of massive haloes decreases in low densities, while the fraction of low mass haloes is similar. The same effect can be seen in the bottom panel of this figure, where we show the PDF of $\bar{\delta}$ for haloes in different mass bins. We find that massive haloes (i.e $m>10^{14} h^{-1} M_{\odot}$ ) reside almost exclusively in very dense regions and are unlikely to be found in regions with low density, while low-mass haloes can be found in a wide range of densities, with preference to average values of $\bar{\delta}=0$.

The dependence of the $b_{\mathrm{h}}(m)$ measurements on the local halo background density $\bar{\delta}$ is in agreement with Abbas \& Sheth (2007). This result is to be expected, since $\bar{\delta}$ is defined from larger scales than $m$, and larger scales are expected to better determine bias. The standard HOD model assumes that halo bias is determined solely by the halo mass, but the local density contains additional information that constrains bias. We therefore test the accuracy of mHOD reconstructions in the following subsection and compare it to reconstructions from our new dHOD model from Eq. (6), which is based on the assumption that the bias is determined solely by $\bar{\delta}$, as discussed in Sect. 2 and suggested by the results of this subsection.

\subsection{HOD tests using mass and density}

In this subsection we study how well mass $m$ and local density $\bar{\delta}$ determine the linear bias of a given halo sample. We do this by testing how well the mHOD model can predict the halo bias as a function of background density $\bar{\delta}$ and how well the dHOD model can predict the halo bias as a function of halo mass $m$. The reconstruction for $b_{\mathrm{h}}(P=\bar{\delta})$ from the mHOD model is derived by averaging the $b_{\mathrm{h}}(m)$ measurement using the measured $\left\langle N_{\mathrm{h}}(m \mid \bar{\delta})\right\rangle$ as weight, as given by Eq. (5). This reconstruction is then compared with measurements of $b_{\mathrm{h}}(\bar{\delta})$, derived from the two-point correlations via Eq. (3). The dHOD reconstructions for $b_{\mathrm{h}}(P=m)$ is tested in an analogous way using $b_{\mathrm{h}}(\bar{\delta})$ and $\left\langle N_{\mathrm{h}}(\bar{\delta} \mid m)\right\rangle$ measurements in combination with Eq. (6).

We can see in the top panel of Fig. 6 that the HMF depends on $\bar{\delta}$ in the high mass end only, while in the low mass end, changes in $\bar{\delta}$ lead to relatively small fluctuations in HMF. Since the low mass end of the HMF dominates the integral of the mHOD bias reconstruction $b_{\mathrm{rec}}^{m}(\bar{\delta})$ from Eq. (5), we do not expect $b_{\text {rec }}^{m}(\bar{\delta})$ to be strongly dependent on $\bar{\delta}$. The PDF of $\bar{\delta}$ in the bottom panel of Fig. 6 shows that haloes of different $m$ are differently distributed in $\bar{\delta}$. Therefore we expect a strong dependence of $b_{\text {rec }}^{\bar{\delta}}(m)$ from Eq. (6) on $m$.

The bias reconstructions $b_{\mathrm{rec}}^{m}(\bar{\delta})$ and $b_{\mathrm{rec}}^{\bar{\delta}}(m)$ are compared with the measured bias $b_{\mathrm{h}}(\bar{\delta})$ and $b_{\mathrm{h}}(m)$ in Fig. 7. As expected from our considerations above, the $b_{\text {rec }}^{m}(\bar{\delta})$ reconstruction does 

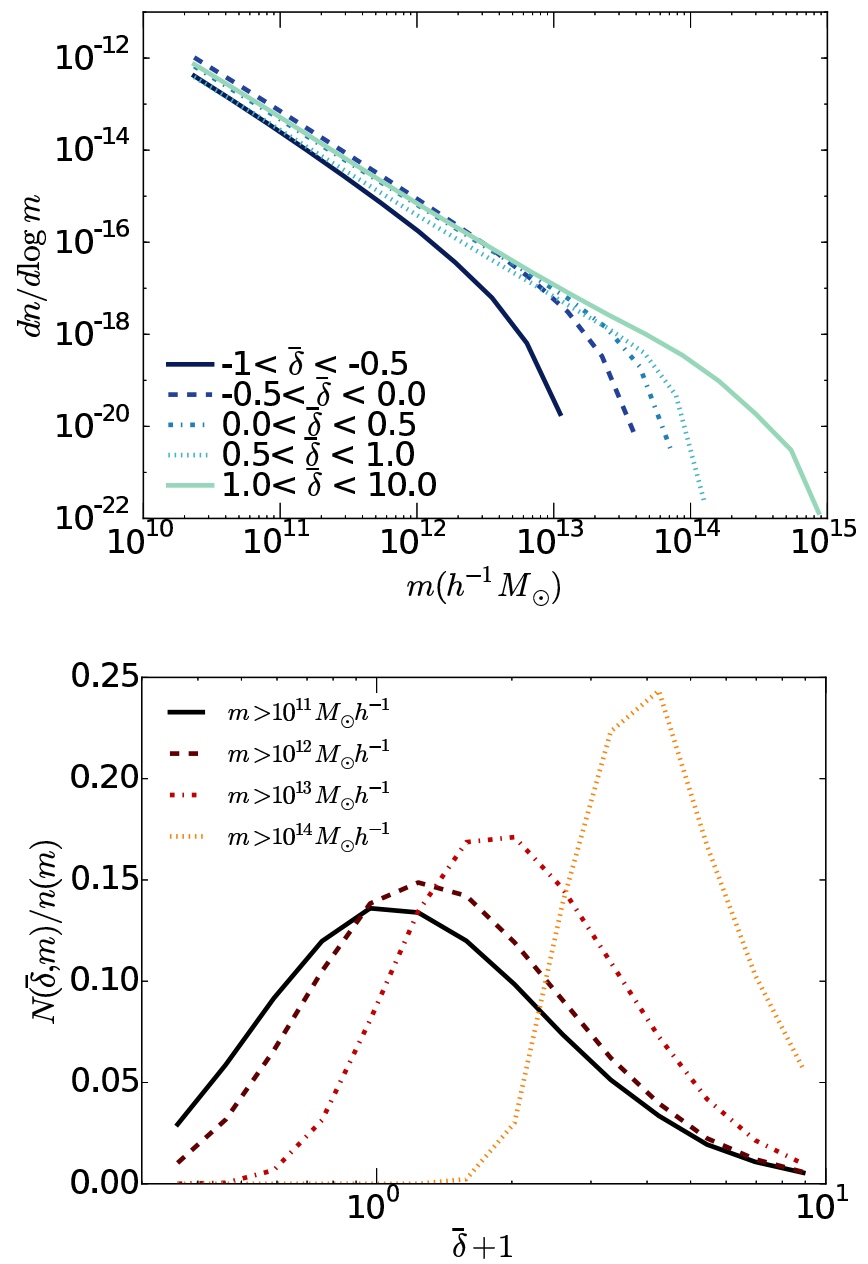

Fig. 6. Distributions of haloes in $m$ and $\bar{\delta}$. Top panel shows the HMF for haloes in different densities. Each colour represents haloes in a range of $\bar{\delta}$. The bottom panel shows the PDF of $\bar{\delta}$ of haloes of different masses. Different colours represent haloes of different $m$ ranges.

not show a significant $\bar{\delta}$ dependence and therefore it deviates from the measured $b_{\mathrm{h}}(\bar{\delta})$. This finding means that, once $\bar{\delta}$ is fixed, the halo mass (or peak height) does not contain additional information about the large-clustering, which already became apparent in the weak mass dependence of the bias of haloes with fixed $\bar{\delta}$, shown in Fig. 4. Hence, the clustering of a given halo sample selected by $\bar{\delta}$ cannot be reconstructed via the standard mHOD model.

The $b_{\mathrm{h}}(m)$ dHOD reconstruction, $b_{\mathrm{rec}}^{\bar{\delta}}(m)$, shown in the bottom panel of Fig. 7, is in much better agreement with the measurement. This finding demonstrates that the bias is accurately determined by $\bar{\delta}$. The over prediction of $b_{\mathrm{h}}$ by the dHOD model at high halo masses results from the fact that $b_{\mathrm{h}}$ is not completely independent of the mass at fixed $\bar{\delta}$, as we see in the left panel of Fig. 4.

\subsection{HOD modelling of galaxy bias}

The results presented in the previous subsection confirm the peak-background split model and have important implications on HOD modelling of galaxy clustering as galaxy properties are not only determined by the mass of their host halo, but also by their interaction with the environment.
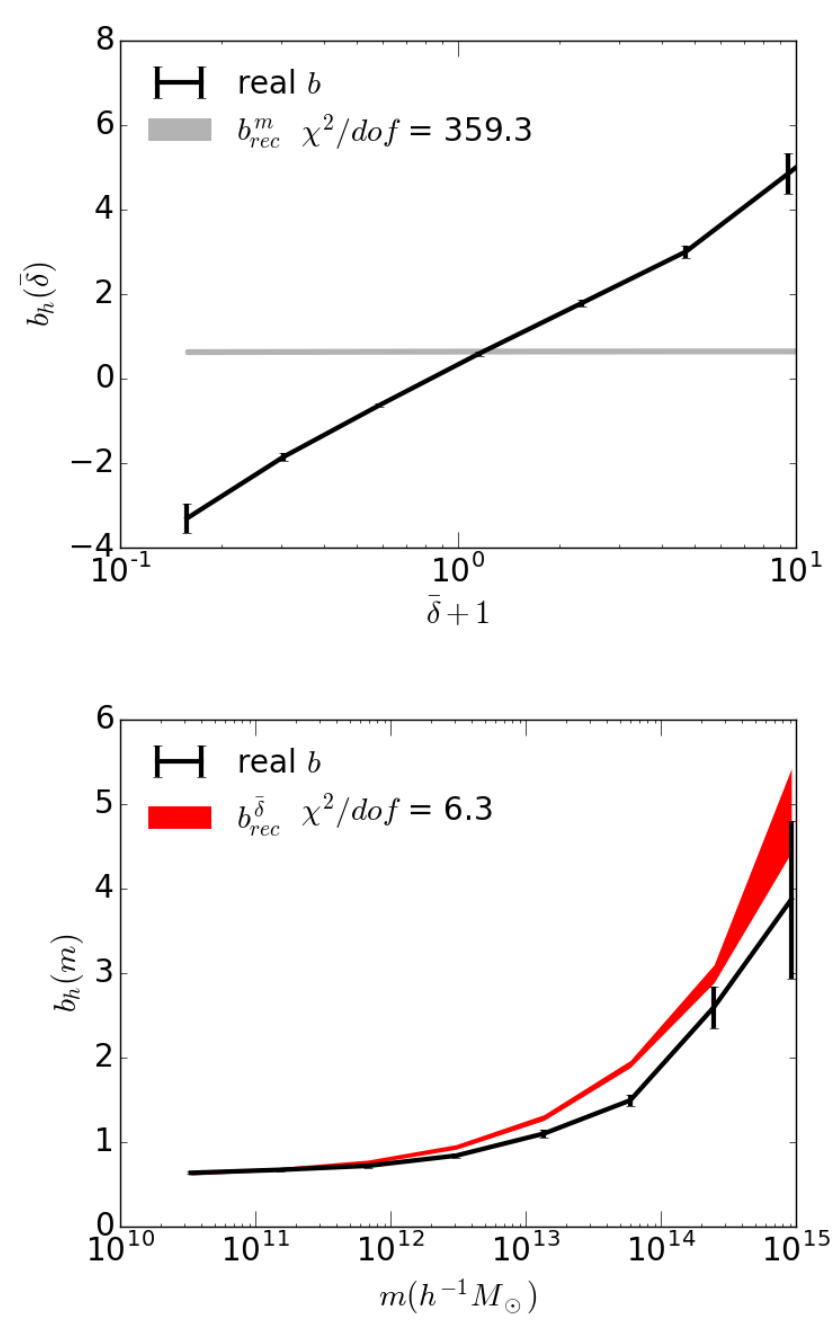

Fig. 7. Halo bias reconstructions compared to the measured bias from the simulation. The solid black lines show the measurement of $b_{\mathrm{h}}$ from the simulation. The coloured regions show the $1 \sigma$ interval of the reconstructions. The top panel shows $b_{\text {rec }}^{m}(\bar{\delta})$ (in grey) from $b_{\mathrm{h}}(m)$, while the bottom panel shows $b_{\mathrm{rec}}^{\bar{\delta}}(m)$ (in red) from $b_{\mathrm{h}}(\bar{\delta})$.

Assuming that bias solely depends on the halo mass can lead to a misinterpretation of HOD predictions (e.g. the fraction of red satellites) and false predictions of galaxy bias as a function of galaxy properties, such as colour or luminosity (Pujol \& Gaztañaga 2014; Zentner et al. 2014). In these cases, it can be worthwhile to use the local density for HOD bias predictions, since we have shown that it determines the bias better than halo mass.

In Fig. 8 we show the comparison of the different HOD reconstruction methods in two different samples of central galaxies from the Guo et al. (2011) SAM. In this analysis we focus on central galaxies, since their properties are more correlated with the halo properties than satellite or orphans galaxies, and the implications of the local density dependence of halo bias are more directly connected to this population.

The top panel shows the bias of central galaxies as a function of the colour index $g-r=M_{\mathrm{g}}-M_{r}$, where $M_{\mathrm{g}}$ and $M_{r}$ are the absolute magnitudes in the SDSS $g$ - and $r$-band, taking into account dust extinction. The bias values below unity result from the fact that we did not apply a faint magnitude cut to the samples. They are therefore dominated by dim galaxies with low clustering. The bottom panel shows the bias of red central galaxies 
A. Pujol et al.: What determines large scale galaxy clustering: halo mass or local density?
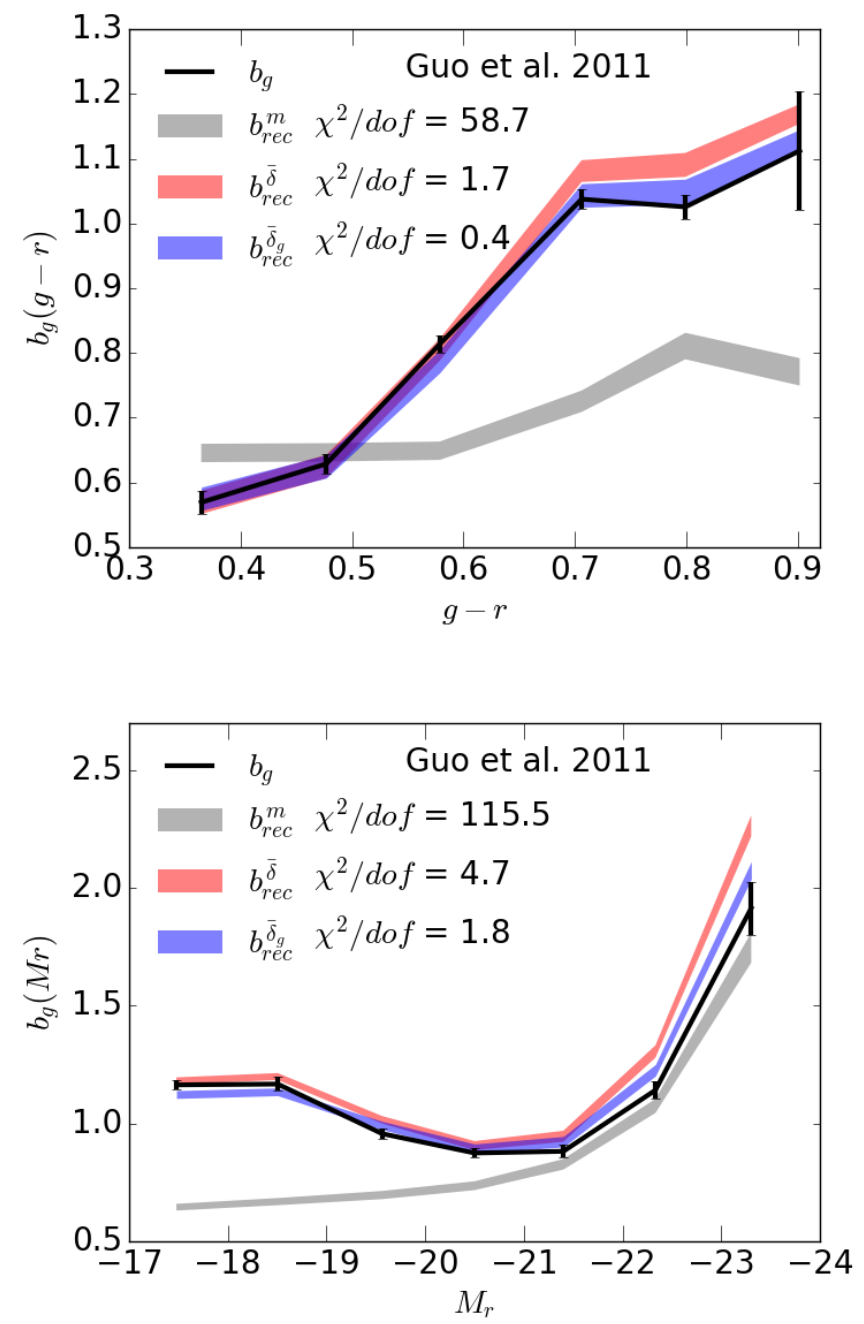

Fig. 8. Galaxy bias compared to different reconstructions. The black solid lines show the measurements of $b_{\mathrm{g}}$ from the simulation. The coloured regions show the $1 \sigma$ level of the different reconstructions of $b_{\mathrm{g}}$ from $b_{\mathrm{h}}(m)$ (so $b_{\mathrm{rec}}^{m}$, in grey), from $b_{\mathrm{h}}(\bar{\delta})$ (so $b_{\mathrm{rec}}^{\bar{\delta}}$, in red) and from $b_{\mathrm{h}}\left(\bar{\delta}_{\mathrm{g}}\right)$ (so $b_{\mathrm{rec}}^{\bar{\delta}_{\mathrm{g}}}$, in blue). The top panel $b_{\mathrm{g}}$ is for central galaxies as a function of colour $g-r$, and in the bottom panel $b_{\mathrm{g}}$ is displayed as a function of absolute $r$-band magnitude for red central galaxies (red defined as $g-r>0.6$ ).

$(g-r>0.6)$ versus $M_{r}$. The solid black line shows the galaxy bias $b_{\mathrm{g}}$ measured from the two-point correlation via Eq. (3). The grey regions show the $1 \sigma$ interval of the $\mathrm{mHOD}$ reconstruction for $b_{\mathrm{g}}$ from Eq. (5), that indicates how well $m$ determines bias for these galaxy samples. The red regions show the $1 \sigma$ interval of the dHOD reconstruction of $b_{\mathrm{g}}$ from Eq. (6), that reflects how well $\bar{\delta}$ determines bias for these galaxies.

We can clearly see that the $\mathrm{dHOD}$ reconstruction is much closer to the measured bias than reconstruction from the mHOD model. This finding indicates that $\bar{\delta}$ determines $b_{\mathrm{g}}$ much better than $m$. This is related to the formation times of the haloes and their assembly history. In terms of galaxy formation, SAMs show that galaxy colours are affected by merging events (Jiménez et al. 2011, and references therein) which occur more often in high density environments. We conclude that, when galaxy properties are determined by halo local density, the standard mHOD model can fail to predict the bias as a function of that property. In this case, HOD bias predictions based on the more fundamental halo property $\bar{\delta}$, that is, the dHOD model, deliver more accurate bias predictions. This effect is not always as important as in Fig. 8, as we can see from Pujol \& Gaztañaga (2014). In this work, $b_{\mathrm{g}}$ is shown as a function of absolute magnitude, and since magnitude and mass are well related for these galaxies, the mHOD reconstruction works well.

In real galaxy catalogues it is very difficult to measure the mean matter density, $\bar{\delta}$, that we are using to define environment. Instead we can easily measure $\bar{\delta}_{\mathrm{g}}$, the density fluctuations of galaxies. In Fig. 8 we also show the bias reconstruction using $\bar{\delta}_{\mathrm{g}}$ instead of $\bar{\delta},\left(b_{\mathrm{rec}}^{\bar{\delta}_{\mathrm{g}}}\right)$, represented as blue regions. We measure $\bar{\delta}_{\mathrm{g}}$ from galaxies of $M_{r}<-19$, and clearly see that the result is equivalent to that using $\bar{\delta}$; in fact it is even better. This means that $\bar{\delta}_{\mathrm{g}}$ determines bias in a similar way to, or better than, $\bar{\delta}$. This result is to be expected as large scale fluctuations in $\bar{\delta}$ and $\bar{\delta}_{\mathrm{g}}$ are simply related to each other by the linear bias of the background galaxy sample. Also note that $\bar{\delta}_{\mathrm{g}}$ is more closely related to the galaxy distribution than $\bar{\delta}$ (the relation between $\bar{\delta}_{\mathrm{g}}$ and $\bar{\delta}$ might have stochasticity, for example) so it is not surprising that $\bar{\delta}_{\text {g }}$ gives a slightly better reconstruction than $\bar{\delta}$. It is important to mention that different galaxy formation models generally present different galaxy distributions. Because of this, the relation between galaxy bias and halo mass or local density can be different depending on the implemented galaxy formation model. For galaxy formation models based on the HOD, constructed using only the halo mass to define the galaxy populations, we would expect, by construction, a better agreement between $b_{\mathrm{g}}$ and $b_{\mathrm{rec}}^{m}$. However, these HOD models would be then totally insensitive to assembly bias effects. This is not the case for SAMs, since these models are constructed from the halo merger trees. This study has been done using four different SAMs in order to see how much our conclusions depend on different implementations of SAMs. We have seen that all SAMs present the same behaviour, and we show the comparison of these models in Appendix A.

\section{Conclusions}

In this paper we use the Millennium simulation (Springel et al. 2005) and their public catalogues to study the impact of halo mass and local density on the prediction of linear bias.

We study the dependence of halo bias on mass (FOF mass) and local density, defined as the density fluctuation $\bar{\delta}$ within a given volume around each halo. Although for this paper we used a cubical box of side $l=14 h^{-1} \mathrm{Mpc}$ (which has the same volume as a sphere of radius $R=8.68 h^{-1} \mathrm{Mpc}$ ), we verified that the results are similar if we use other scales. We also find similar results when we use Lagrangian instead of Eulerian densities for the background.

We show that bias depends much more strongly on $\bar{\delta}$ than on mass, and once $\bar{\delta}$ is fixed, the halo bias depends very weakly on mass. This is important, since it reflects the fact that the halo bias is well constrained when the haloes are selected by the local density, almost independently from the mass. More massive haloes have a higher clustering because, statistically, they are in denser regions, but not because mass is the fundamental cause of clustering. In particular, low-mass haloes can be found in a wide range of densities, and hence these haloes present different clustering. These results confirm the peak-background split model, that states that the large-scale clustering is determined by the density fluctuations of matter, and not by halo mass. This is in contradiction with the standard HOD implementation which assumes that halo bias only depends on the mass. The local density can be seen as a property that is sensitive to other dependencies 
of halo bias apart from mass, such as assembly bias. Assembly bias usually refers to the difference in clustering from haloes of equal mass but different formation time or concentration. These differences can indeed be related to the local density, as haloes in high background densities form first and have higher concentrations. This can also explain the concept of galactic conformity (see Paranjape et al. 2015, and references therein) by which galaxy properties, such as luminosity and colour, are not solely determined by the mass of the halo. Thus, our finding that halo local density, and not halo mass, is the key variable in understanding the large-scale clustering of haloes is in line with these previous results.

To study the implication of our finding for the clustering predictions in the HOD framework, we use the method of reconstructing the linear bias from the halo bias and the occupation distribution in haloes, as explained in Pujol \& Gaztañaga (2014) and in this paper. This can be seen as a test of how well mass and local density constrain bias. More precisely, these bias reconstructions measure how well linear bias can be reproduced by assuming that the halo bias and occupation only depend on one variable (either mass or density around the halo). We show that we can predict $b_{\mathrm{h}}(M)$ from $b_{\mathrm{h}}(\bar{\delta})$, but we cannot predict $b_{\mathrm{h}}(\bar{\delta})$ from $b_{\mathrm{h}}(M)$. This means that $\bar{\delta}$ determines bias better than $M$. This is important for HOD analysis, since it is usually assumed that bias only depends on halo mass, but some galaxy populations might be affected by environment and local density as well. According to our results, the dependencies of galaxies on the environmental density have a stronger impact on the large scale clustering than the dependencies on halo mass. With the exception of the higher mass range, which is strongly correlated with background density, as shown in Fig. 5, and therefore shows similar tendencies than local density.

Some of the galaxy properties can be sensitive to assembly bias and the local density. In these cases, assuming that linear bias only depends on halo mass causes an error in our estimation of clustering or in the estimation of HOD parameters. Instead, we can use the local density as a proxy for bias, since on large scales it determines bias better than mass.

We show two examples of galaxy samples, $b\left(M_{r}\right)$ of red central galaxies and $b(g-r)$ of central galaxies, where the galaxy clustering does not depend only on halo mass. We see that the reconstruction using the local density $\bar{\delta}$ around the haloes makes a good prediction of galaxy bias, but the standard reconstruction using halo mass does not recover the galaxy bias well. This means, on the one hand, that the occupation of this population of galaxies in haloes is affected by the local density even for fixed mass, and on the other hand, that even if the occupation of these galaxies in haloes depends on mass, the clustering of these galaxies is mainly due to the dependence on the local density. We also used $\bar{\delta}_{\mathrm{g}}$ instead of $\bar{\delta}$ to measure the local density of haloes and the results are equivalent to those using $\bar{\delta}$, meaning that both $\bar{\delta}$ and $\bar{\delta}_{\mathrm{g}}$ are good estimators of bias. This result is to be expected, since at large scales, $\bar{\delta}_{\mathrm{g}}$ is biased with respect to $\bar{\delta}$, but the nature of both properties is the same, and hence they disclose similar information about the local density. The advantage of using $\bar{\delta}_{\mathrm{g}}$ is that it can be directly measured in observations, while $\bar{\delta}$ or halo mass are more difficult to estimate. We also show that our results and conclusions do not depend on the SAM used for the analysis.

This analysis is focused on linear scales, where the 2-halo term dominates, therefore we do not need to assume anything about the distribution of galaxies inside the haloes. A similar analysis is relevant for the 1-halo term, since small scales can also depend on local density. For example, the satellite distribution can depend on the halo concentration for fixed mass. Also, these linear scales now become more accessible with upcoming surveys. As $\bar{\delta}$ is a better estimator of large-scale clustering than mass, and it is also easier to measure in observations (at large scales $\bar{\delta}_{\mathrm{g}}$ is simply a biased version of $\bar{\delta}$ ), this method can be applied in observations to measure the bias as a function of $\bar{\delta}$, and to study galaxy clustering from the modelling of $b_{\mathrm{h}}(\bar{\delta})$ instead of simply $b_{\mathrm{h}}(M)$ in order to incorporate the information from assembly bias and environmental dependencies of bias.

Acknowledgements. We thank Ravi Sheth and Román Scoccimarro for useful discussions about the project. We also thank Sergio Contreras for support and discussions about the Millennium simulation and its database. Funding for this project was partially provided by the Spanish Ministerio de Ciencia e Innovación (MICINN), Consolider-Ingenio CSD2007- 00060, European Commission Marie Curie Initial Training Network CosmoComp (PITNGA-2009-238356). We acknowledge support from the European Commission's Framework Programme 7, through the Marie Curie International Research Staff Exchange Scheme LACEGAL (PIRSES-GA-2010-269264). A.P. is supported by beca FI and 2009-SGR1398 from Generalitat de Catalunya and project AYA2012-39620 from MICINN. During the work on this project K.H. has been supported by beca FI from Generalitat de Catalunya and ESP2013-48274-C3-1-P. He also acknowledges ICTPSAIFR, where parts of the project were done. N.J. acknowledges support from CONICET-Argentina; the European Research Council Starting Grant (SEDmorph; P.I. V. Wild); and the European Commission's Framework Programme 7, through the Marie Curie International Research Staff Exchange Scheme LACEGAL (PIRSES-GA-2010- 269264). Special thanks to Institut de Ciències de l'Espai, for their hospitality.

\section{References}

Abbas, U., \& Sheth, R. K. 2007, MNRAS, 378, 641

Baldauf, T., Seljak, U., Desjacques, V., \& McDonald, P. 2012, Phys. Rev. D, 86, 083540

Bardeen, J. M., Bond, J. R., Kaiser, N., \& Szalay, A. S. 1986, ApJ, 304, 15

Baugh, C. M. 2006, Rep. Prog. Phys., 69, 3101

Bel, J., Hoffmann, K., \& Gaztañaga, E. 2015, MNRAS, 453, 259

Benson, A. J., Cole, S., Frenk, C. S., Baugh, C. M., \& Lacey, C. G. 2000, MNRAS, 311, 793

Berlind, A. A., \& Weinberg, D. H. 2002, ApJ, 575, 587

Bett, P., Eke, V., Frenk, C. S., et al. 2007, MNRAS, 376, 215

Bower, R. G., Benson, A. J., Malbon, R., et al. 2006, MNRAS, 370, 645

Carretero, J., Castander, F. J., Gaztañaga, E., Crocce, M., \& Fosalba, P. 2015, MNRAS, 447, 646

Chan, K. C., Scoccimarro, R., \& Sheth, R. K. 2012, Phys. Rev. D, 85, 083509

Cole, S., \& Kaiser, N. 1989, MNRAS, 237, 1127

Cole, S., Percival, W. J., Peacock, J. A., et al. 2005, MNRAS, 362, 505

Cooray, A. 2006, MNRAS, 365, 842

Cooray, A., \& Sheth, R. 2002, Phys. Rep., 372, 1

Conroy, C., Wechsler, R. H., \& Kravtsov, A. V. 2006, ApJ, 647, 201

Croft, R. A. C., Matteo, T. D., Khandai, N., et al. 2012, MNRAS, 425, 2766

Croton, D. J., Gao, L., \& White, S. D. M. 2007, MNRAS, 374, 1303

De Lucia, G., \& Blaizot, J. 2007, MNRAS, 375, 2

Faltenbacher, A., \& White, S. D. M. 2010, ApJ, 708, 469

Font, A. S., Bower, R. G., McCarthy, I. G., et al. 2008, MNRAS, 389, 1619

Fosalba, P., \& Gaztanaga, E. 1998, MNRAS, 301, 503

Gao, L., \& White, S. D. M. 2007, MNRAS, 377, L5

Gao, L., Springel, V., \& White, S. D. M. 2005, MNRAS, 363, L66

Gargiulo, I. D., Cora, S. A., Padilla, N. D., et al. 2015, MNRAS, 446, 3820

Guo, Q., White, S., Boylan-Kolchin, M., et al. 2011, MNRAS, 413, 101

Harker, G., Cole, S., Helly, J., Frenk, C., \& Jenkins, A. 2006, MNRAS, 367, 1039

Hearin, A. P., Zentner, A. R., Berlind, A. A., \& Newman, J. A. 2013, MNRAS, 433,659

Hearin, A. P., Watson, D. F., Becker, M. R., et al. 2014, MNRAS, 444, 729

Hearin, A. P., Watson, D. F., \& van den Bosch, F. C. 2015, MNRAS, 452, 1958

Hearin, A. P., Zentner, A. R., van den Bosch, F. C., Campbell, D., \& Tollerud, E. 2016, MNRAS, 460, 2552

Hoffmann, K., Bel, J., \& Gaztañaga, E. 2015, MNRAS, 450, 1674

Jiménez, N., Cora, S. A., Bassino, L. P., Tecce, T. E., \& Smith Castelli, A. V. 2011, MNRAS, 417, 785 
A. Pujol et al.: What determines large scale galaxy clustering: halo mass or local density?

Jing, Y. P., Mo, H. J., \& Boerner, G. 1998, ApJ, 494, 1

Jing, Y. P., Suto, Y., \& Mo, H. J. 2007, ApJ, 657, 664

Lacerna, I., \& Padilla, N. 2011, MNRAS, 412, 1283

Lacerna, I., \& Padilla, N. 2012, MNRAS, 426, L26

Lacerna, I., Padilla, N., \& Stasyszyn, F. 2014, MNRAS, 443, 3107

Masaki, S., Lin, Y.-T., \& Yoshida, N. 2013, MNRAS, 436, 2286

Merson, A. I., Baugh, C. M., Helly, J. C., et al. 2013, MNRAS, 429, 556

Mo, H. J., \& White, S. D. M. 1996, MNRAS, 282, 347

Norberg, P., Baugh, C. M., Gaztañaga, E., \& Croton, D. J. 2009, MNRAS, 396, 19

Paranjape, A., Kovac, K., Hartley, W. G., \& Pahwa, I. 2015, MNRAS, 454, 3030

Pujol, A., \& Gaztañaga, E. 2014, MNRAS, 442, 1930

Rodríguez-Torres, S. A., Prada, F., Chuang, C.-H., et al. 2016, MNRAS, 460, 1173

Scoccimarro, R., Sheth, R. K., Hui, L., \& Jain, B. 2001, ApJ, 546, 20

Seljak, U. 2000, MNRAS, 318, 203

Seljak, U., \& Zaldarriaga, M. 1996, ApJ, 469, 437

Sheth, R. K. 1998, MNRAS, 300, 1057
Sheth, R. K., \& Tormen, G. 2004, MNRAS, 350, 1385

Spergel, D. N., Verde, L., Peiris, H. V., et al. 2003, ApJS, 148, 175

Springel, V. 2005, MNRAS, 364, 1105

Springel, V., White, S. D. M., Tormen, G., \& Kauffmann, G. 2001, MNRAS 328,726

Springel, V., White, S. D. M., Jenkins, A., et al. 2005, Nature, 435, 629

Tinker, J., Kravtsov, A. V., Klypin, A., et al. 2008, ApJ, 688, 709

Tinker, J. L., Robertson, B. E., Kravtsov, A. V., et al. 2010, ApJ, 724, 878

Tinker, J. L., Leauthaud, A., Bundy, K., et al. 2013, ApJ, 778, 93

van den Bosch, F. C., Yang, X., Mo, H. J., et al. 2007, MNRAS, 376, 841

Wetzel, A. R., Cohn, J. D., White, M., Holz, D. E., \& Warren, M. S. 2007, ApJ, 656,139

White, S. D. M., \& Rees, M. J. 1978, MNRAS, 183, 341

Wu, H.-Y., Rozo, E., \& Wechsler, R. H. 2008, ApJ, 688, 729

Yang, X., Mo, H. J., \& van den Bosch, F. C. 2003, MNRAS, 339, 1057

Zehavi, I., Zheng, Z., Weinberg, D. H., et al. 2005, ApJ, 630, 1

Zehavi, I., Zheng, Z., Weinberg, D. H., et al. 2011, ApJ, 736, 59

Zentner, A. R., Hearin, A. P., \& van den Bosch, F. C. 2014, MNRAS, 443, 3044

Zheng, Z., Coil, A. L., \& Zehavi, I. 2007, ApJ, 667, 760 


\section{Appendix A: Comparison of different semi-analytic models}

In this appendix we show the results of our analysis for three different SAMs in order to compare them with the results from Fig. 8 from the Guo et al. (2011) model. In Fig. A.1 we show the same results for the Bower et al. (2006) model (top panels), De Lucia \& Blaizot (2007) model (middle panels) and Font et al. (2008) model (bottom panels). As in Fig. 8, the measurements of $b_{\mathrm{g}}$ are shown as solid black lines, and the errors represent the $1 \sigma$ level. The coloured regions show $b_{\text {rec }}^{m}$ (in grey), $b_{\text {rec }}^{\bar{\delta}}$ (in red) and $b_{\text {rec }}^{\bar{\delta}_{\mathrm{g}}}$ (in blue) at the $1 \sigma$ level. The left panels show the bias reconstructions for central galaxies as a function of $g-r$ colour, while the right panels show red central galaxies as a function of luminosity $\left(M_{r}\right)$. The colour cut applied corresponds, as in Fig. 8, to $g-r>0.6$.

We can see that the results from De Lucia \& Blaizot (2007) and Guo et al. (2011) are very similar. The same happens between Bower et al. (2006) and Font et al. (2008). This result is to be expected because of other similarities between De Lucia \& Blaizot (2007) and Guo et al. (2011; and Bower et al. 2006 and Font et al. 2008). In particular, De Lucia \& Blaizot (2007) and Guo et al. (2011) follow the merger trees according to the subhalo catalogues obtained from SUBFIND (Springel et al. 2001). On the other hand, Bower et al. (2006); and Font et al. (2008) follow the merger trees from the Dhaloes, a different definition of haloes obtained from the contribution of several SUBFIND sub-haloes (Harker et al. 2006; Merson et al. 2013). As De Lucia \& Blaizot (2007) and Guo et al. (2011) have a common merger tree, their statistics, and in particular their clustering, are very similar (the same effect occurs for Bower et al. 2006; and Font et al. 2008). However, the differences between the models are larger when we compare models with different merger trees. For the rest of the discussion we will refer to De Lucia \& Blaizot (2007) and Guo et al. (2011) as MPA models, and to Bower et al. (2006) and Font et al. (2008) as Durham models, according to the institutes where they were developed.

We see some interesting differences between MPA and Durham models. If we focus on the left plots, where we compare different reconstructions of galaxy bias as a function of colour, we see that Durham models present a better agreement between $b_{\mathrm{g}}$ and $b_{\mathrm{rec}}^{m}$ than MPA models. This means that halo mass better constrains galaxy bias for Durham models than for MPA models. This is an example of how the agreements of the reconstructions can depend on the implementation of the galaxy formation model and the employed merger tree. Another important difference is on the right plots, where we show galaxy bias of red central galaxies as a function of luminosity. We can see that dim galaxies of MPA models present a strong clustering, while Durham models do not. The low values of $b_{\text {rec }}^{m}$ imply that these galaxies populate small haloes in all the models. But although the masses are similar, MPA models populate different haloes than Durham models, since the clustering is very different. In particular, red dim galaxies of MPA models populate small haloes that present a very high clustering, showing a bias of up to 1.4. However, dim red galaxies of Durham models show a bias below 1 in all the cases.

However, although the different SAMs can present different numbers, it is important to notice that the qualitative conclusions of this study are common in all the SAMs. First of all, in all the cases, mass is always the least constraining variable on galaxy bias. In all the cases, $b_{\text {rec }}^{\bar{\delta}}$ and $b_{\text {rec }}^{\bar{\delta}_{\mathrm{g}}}$ show very similar results, while $b_{\text {rec }}^{\bar{\delta}_{\mathrm{g}}}$ works slightly better. Because of this, our discussion and conclusions from Fig. 8 are valid for all the SAMs studied. 
A. Pujol et al.: What determines large scale galaxy clustering: halo mass or local density?
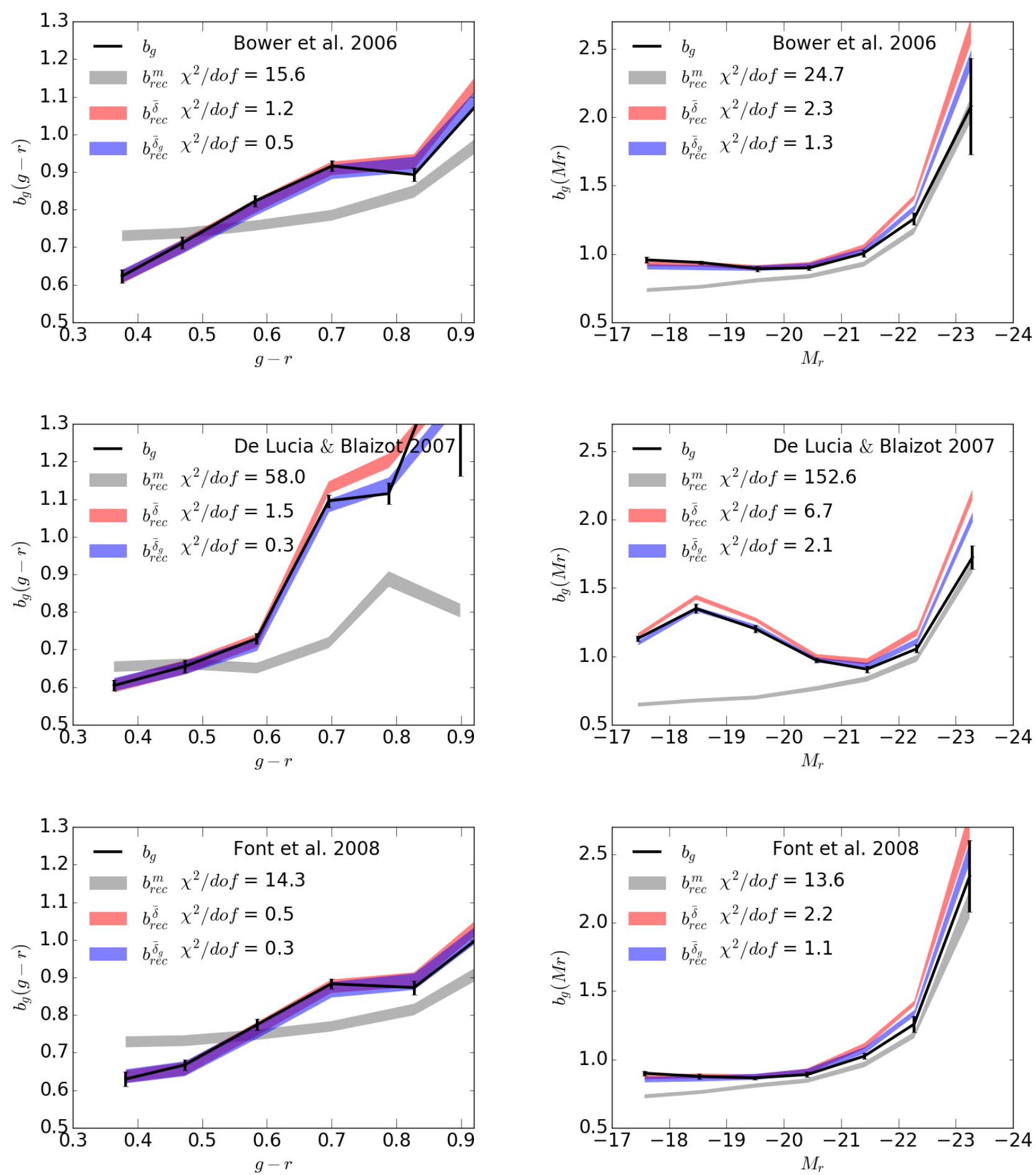

Fig. A.1. Comparison of bias reconstructions for different SAMs. The top panels show the results for Bower et al. (2006) model, middle panels represent De Lucia \& Blaizot (2007) model and Font et al. (2008) is shown in the bottom panels. As in Fig. 8, black solid lines show the measurements of $b_{\mathrm{g}}$, while $b_{\text {rec }}^{m}, b_{\text {rec }}^{\bar{\delta}}$ and $b_{\text {rec }}^{\bar{\delta}_{\mathrm{g}}}$ are shown in grey, red and blue, respectively. Left panels show central galaxies as a function of colour $g-r$, while right panels show red $(g-r>0.6)$ central galaxies as a function of $r$-band absolute luminosity. 